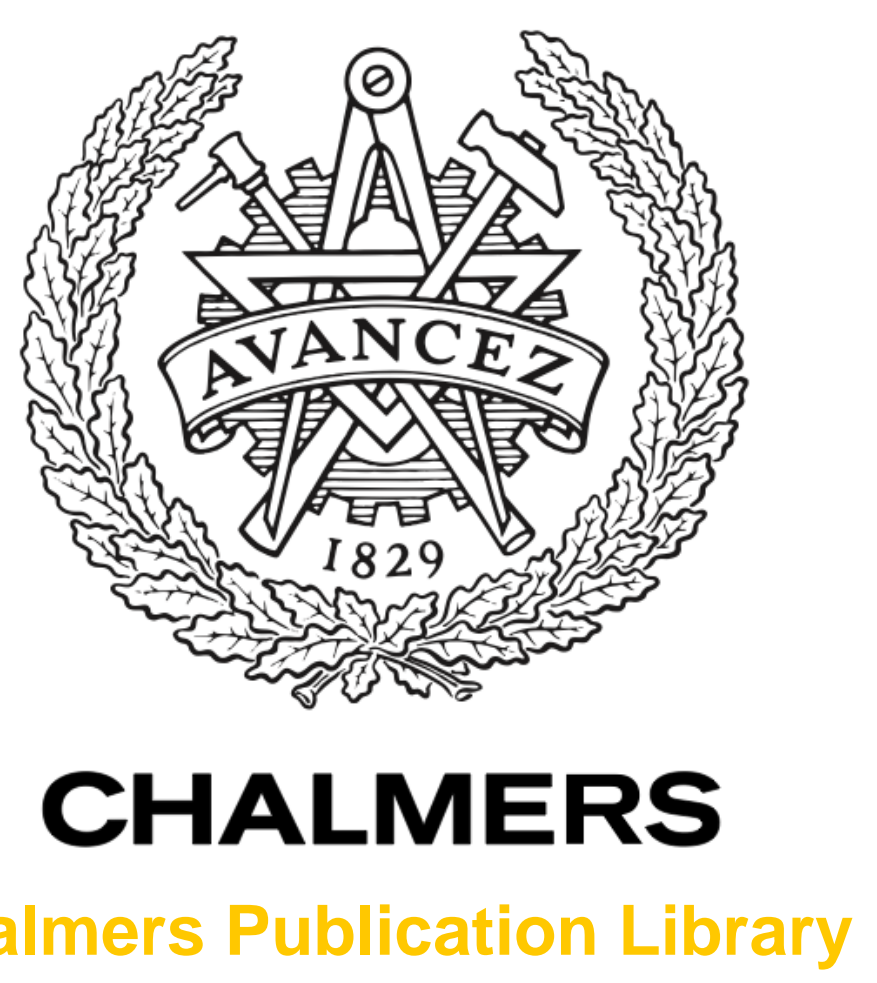

Chalmers Publication Library

Revisiting the Complex Correlation in a MIMO System

This document has been downloaded from Chalmers Publication Library (CPL). It is the author's version of a work that was accepted for publication in:

8th European Conference on Antennas and Propagation, EuCAP 2014, The Hague, The Netherlands 6-11 April 2014

Citation for the published paper:

Chen, X. ; Kildal, P. ; Carlsson, J. (2014) "Revisiting the Complex Correlation in a MIMO System". 8th European Conference on Antennas and Propagation, EuCAP 2014, The Hague, The Netherlands 6-11 April 2014 pp. 2735 - 2739.

http://dx.doi.org/10.1109/EuCAP.2014.6902390

Downloaded from: http://publications.lib.chalmers.se/publication/203781

Notice: Changes introduced as a result of publishing processes such as copy-editing and formatting may not be reflected in this document. For a definitive version of this work, please refer to the published source. Please note that access to the published version might require a subscription.

Chalmers Publication Library (CPL) offers the possibility of retrieving research publications produced at Chalmers University of Technology. It covers all types of publications: articles, dissertations, licentiate theses, masters theses, conference papers, reports etc. Since 2006 it is the official tool for Chalmers official publication statistics. To ensure that Chalmers research results are disseminated as widely as possible, an Open Access Policy has been adopted.

The CPL service is administrated and maintained by Chalmers Library. 


\title{
Revisiting the Complex Correlation in a MIMO System
}

\author{
Xiaoming Chen ${ }^{1}$, Per-Simon Kildal ${ }^{2}$, Jan Carlsson ${ }^{3}$ \\ 123 Department of Signals and Systems, Chalmers University of Technology, Gothenburg, Sweden, xiaoming.chen@chalmers.se \\ ${ }^{3}$ Electronics Department, SP Technical Research Institute of Sweden, Borås, Sweden
}

\begin{abstract}
It is commonly believed that a complex correlation of up to 0.5 in magnitude is negligible for multiple-input multipleoutput (MIMO) performances and that the correlation affects the diversity gain or throughput only via its magnitude. In this work, we show that when the number of antenna ports is larger than two, the phase of the correlation coefficient affects the diversity gain and that even small correlation can severely degrade the throughput performance of MIMO systems with full spatial multiplexing and no diversity gain. We assume maximum ratio combining (MRC) to investigate the correlation effect on diversity gain and zero-forcing (ZF) equalizer to study the correlation effect on the MIMO throughput.
\end{abstract}

Index Terms-Correlation, diversity, multiple-input multipleouput (MIMO), maximum ratio combining (MRC), throughput, zero-forcing (ZF).

\section{INTRODUCTION}

Multiple-input multiple-output (MIMO) systems have attracted lots of attentions during the past decades, by virtue of their abilities of increasing the communication reliability (via diversity techniques) and the data rate (via spatial multiplexing) [1]. A MIMO system necessitates multi-port antennas, but in practice, the space allocated for the multi-port antenna in the MIMO system is usually limited. Hence, compact multi-port antennas are desired. However, a drawback of using compact multi-port antennas is that the (spatial) correlation and the mutual coupling become inevitable [2], [3]. This paper focuses on the effect of correlation on MIMO systems.

It is commonly believed that a complex correlation of up to 0.5 in magnitude is negligible for MIMO performances (see e.g., [4]) and that the correlation affects the MIMO performances only via its magnitude (e.g., [5]). In this work, however, we will show that the degradation due to correlations on the MIMO performance depends on the number of antenna ports. We will also show that for a two-port antenna, the correlation affects MIMO performances only via its magnitude, whereas for antennas with more than two ports, the phase of the correlation also affects the MIMO performance. For instance, two correlations with the same magnitude but with opposite phases may result in a big difference in the throughput performance ${ }^{1}$.

\footnotetext{
${ }^{1}$ We will give a proof of this in a sequel of this paper.
}

In this paper, we revisit the complex correlation and its effects on diversity and throughput performances. For the diversity performance evaluation, we assume that the multiport antenna (or the diversity antenna) uses the maximum ratio combining (MRC) technique. For the throughput performance evaluation, we assume an open-loop ${ }^{2}$ MIMO system with a zero-forcing (ZF) receiver [1]. Albeit not achievable in practice (due to the requirements of input signals with Gaussian distribution and infinite sets of modulation and coding schemes), capacity has been a popular metric for MIMO performance. Hence, we also characterize the MIMO performance using capacity in this paper.

\section{MIMO PERFORMANCE IN CORRELATED CHANNEL}

\section{A. Diversity Gain}

Assuming an $\mathrm{N}$-port diversity antenna in a Rayleigh-fading environment, the correlation matrix is

$$
\mathbf{R}=E\left[\mathbf{h h}^{H}\right]
$$

where $\mathbf{h}$ is the column-vector diversity channel including the overall antenna effect, the superscript ${ }^{H}$ is the Hermitian operator, and $E$ is the expectation. The output power of the MRC combiner is $P_{M R C}=\mathbf{h}^{H} \mathbf{h}$. Assuming independent, identically distributed (i.i.d.) Gaussian noises with unity variance, $P_{M R C}$ then equals in value to the instantaneous signal-to-noise ratio (SNR), denoted as $\gamma$. The diversity gain (DG) is defined as the output SNR of a diversity antenna relative to that of a single ideal (i.e. with $100 \%$ total radiation efficiency) antenna at certain outage probability level, e.g. $1 \%$ [6], [7]. The MRC DG is then

$$
D G=F^{-1}(\gamma) /\left.F_{\text {ideal }}^{-1}(\gamma)\right|_{1 \%}
$$

where $(\cdot)^{-1}$ denotes functional inversion, $F$ is the cumulative distribution function of the MRC output SNR, and $F_{\text {ideal }}(\gamma)=1-\exp (-\gamma)$ is the CDF of the output SNR of the ideal reference antenna in the Rayleigh fading environment.

The CDF of the MRC output SNR in Rayleigh fading is known for two cases:

\footnotetext{
2 In an open-loop MIMO system, the channel state information (CSI) estimated by the receiver will not be feedback to the transmitter. So the receiver knows the CSI, whereas the transmitter does not.
} 


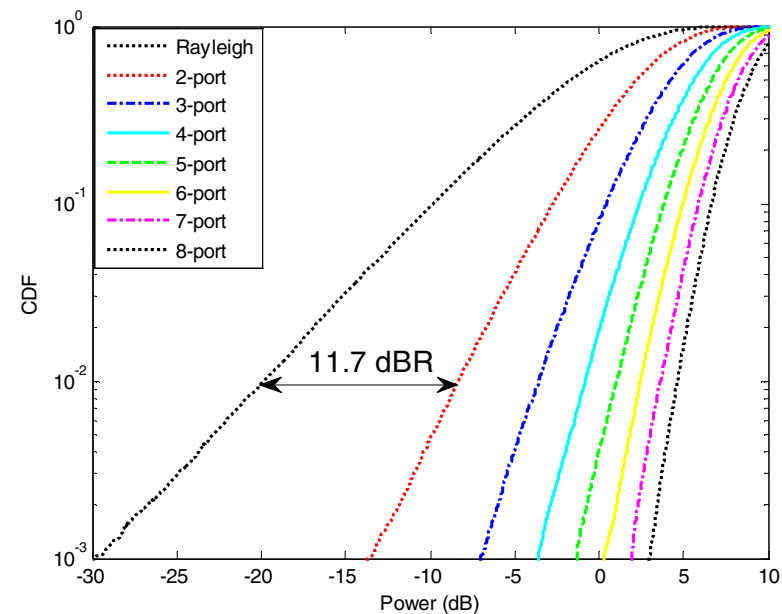

Fig. 1. CDF of Rayleigh and MRC output for the i.i.d. case.
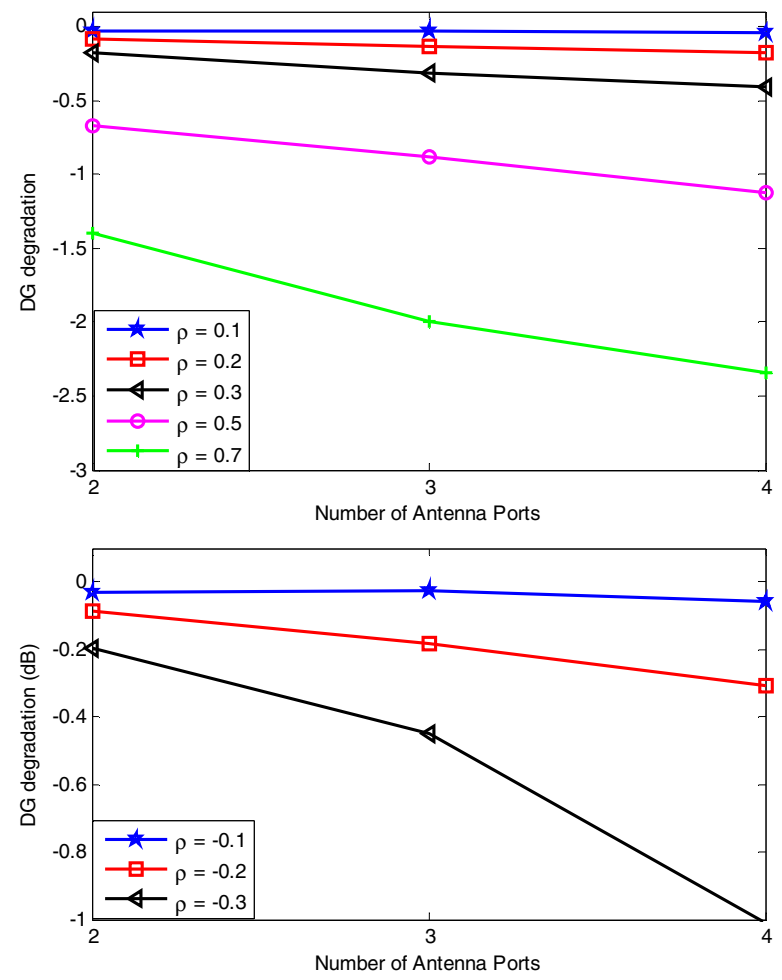

Fig. 2. DG degradation due to uniform correlation with respect to the i.i.d. channel case. Upper graph corresponds to positive correlation; lower graph corresponds to negative correlation.

- When all eigenvalues are equal, i.e. $\lambda_{i}=\lambda(i=1 \ldots$ $N)$, it is given by [6]

$$
F(\gamma)=1-\exp (-\gamma / \lambda) \sum_{i=1}^{N}(\gamma / \lambda)^{i-1} /(i-1) !
$$

- When all eigenvalues are different from each other, it is given by [8]

$$
F(\gamma)=1-\sum_{i=1}^{N} \lambda_{i}^{N-1} \exp \left(-\gamma / \lambda_{i}\right) / \prod_{k \neq i}^{N}\left(\lambda_{i}-\lambda_{k}\right)
$$

where $\lambda_{i}$ denotes the $i$ th eigenvalues of $\mathbf{R}$. The CDF expressions with arbitrary equal eigenvalues are unknown in general and have to be approximated by empirical CDFs from measured channel samples. For generality, we obtain the empirical CDF by numerical simulation. The CDF expressions (3) and (4) are useful in the analysis.

Fig. 1 shows the CDFs of the Rayleigh and the MRC output SNRs of different multi-port antennas in i.i.d. Rayleigh channels. For illustration of the DG definition, the MRC DG of a two-port antenna in the i.i.d. Rayleigh channel is marked in Fig. 1.

To examine the effect of the correlation on the DG, we define the DG degradation as the ratio of DG in the presence of correlation to the corresponding DG in the i.i.d. channel. Fig. 2 shows the DG degradation for the special cases of positive and negative uniform correlations among antenna ports, respectively. Note that the assumption of a uniform correlation of -0.3 is valid up to four-port antenna only. Beyond four ports, the corresponding "correlation matrix" will not be positive semi-definite [9], which means it is nonphysical. It can be seen that the DG degradation due to correlation is larger for the diversity antenna with more ports and that the negative correlation causes more DG degradation than the positive correlation. Hence, the phase of the correlation also affects the diversity performance.

Before giving an explanation of this, we show first that, for a two-port antenna $(N=2)$, the DG depends only on the magnitude of the correlation:

In a two-port antenna, the CDF of the MRC output SNR (4) reduces to

$$
F(\gamma)=1-\frac{\lambda_{1} \exp \left(-\gamma / \lambda_{1}\right)-\lambda_{2} \exp \left(-\gamma / \lambda_{2}\right)}{\lambda_{1}-\lambda_{2}} .
$$

It was shown in [10] that (5) converges to the true value as $\lambda_{i} \rightarrow \lambda(i=1,2)$. (The two eigenvalues are equal for powerbalanced two-port antenna without correlation.) The eigenvalues of the covariance matrix

$$
\mathbf{R}=\left[\begin{array}{cc}
1 & \rho \\
\rho * & 1
\end{array}\right]
$$

are

$$
\begin{aligned}
& \lambda_{1}=1+|\rho| \\
& \lambda_{2}=1-|\rho|
\end{aligned}
$$

where $\rho$ denotes the complex-valued correlation between the two antenna ports and the superscript $*$ denotes the complex conjugate. Combining (2), (5), and (7), it follows that the DG of the two-port antenna depends only on the correlation magnitude. This completes the proof.

From (2) and (4), it is obvious that the DG depends on the eigenvalues of the correlation matrix $\mathbf{R}$. Before we show why the phase of the correlation affects the DG for antennas with more than two ports, some prerequisites are needed for the majorization theory.

Majorization [11]: For two real-valued $M \times 1$ vectors a and $\mathbf{b}$ in descending order, a majorizes $\mathbf{b}$, denoted as $\mathbf{a} \succ \mathbf{b}$, if $\sum_{i=1}^{m} a_{i} \geq \sum_{i=1}^{m} b_{i} \quad(m=1, \ldots, M-1)$ and $\sum_{i=1}^{M} a_{i}=\sum_{i=1}^{M} b_{i}$. Assume that $\mathbf{a}$ and $\mathbf{b}$ are two eigenvalue vectors associated 
with correlation matrices $\mathbf{R}_{a}$ and $\mathbf{R}_{b}, \mathbf{R}_{a}$ is more correlated than $\mathbf{R}_{b}$ if $\mathbf{a} \succ \mathbf{b}$.

For two correlation matrices with the same correlation magnitudes but different correlation phases, their eigenvalue vectors are different. (Note that throughout this paper without loss of generality eigenvalue vectors are assumed to be in descending order.) We give a numerical example in the sequel for better illustration.

For a correlation matrix of a four-port antenna with uniform correlation of 0.3 , i.e.,

$$
\mathbf{R}_{a}=\left[\begin{array}{cccc}
1 & 0.3 & 0.3 & 0.3 \\
0.3 & 1 & 0.3 & 0.3 \\
0.3 & 0.3 & 1 & 0.3 \\
0.3 & 0.3 & 0.3 & 1
\end{array}\right],
$$

the eigenvalue vector is $\mathbf{a}=\left[\begin{array}{llll}1.9 & 0.7 & 0.7 & 0.7\end{array}\right]^{T}$; for a correlation matrix of a four-port antenna with uniform correlation of -0.3 , i.e.,

$$
\mathbf{R}_{b}=\left[\begin{array}{cccc}
1 & -0.3 & -0.3 & -0.3 \\
-0.3 & 1 & -0.3 & -0.3 \\
-0.3 & -0.3 & 1 & -0.3 \\
-0.3 & -0.3 & -0.3 & 1
\end{array}\right],
$$

the eigenvalue vector is $\mathbf{b}=\left[\begin{array}{llll}1.3 & 1.3 & 1.3 & 0.1\end{array}\right]^{T}$. Hence, $\mathbf{b} \succ \mathbf{a}$ and $\mathbf{R}_{b}$ is more correlated than $\mathbf{R}_{a}$. This explains why the DG of a four-port antenna with a uniform correlation of -0.3 is smaller than that with a uniform correlation of 0.3 (see Fig. 2).

\section{B. Throughput}

Throughput is the actual data rate. A simple throughput model has been presented in [12], [13], based on which, the average throughput of a system with fixed modulation and coding scheme (MCS) in a fading channel can be approximated by

$$
T_{\text {put }}(\bar{\gamma})=T_{\text {put,max }}\left(1-F\left(\gamma_{t h} / \bar{\gamma}\right)\right)
$$

where $\bar{\gamma}$ represents the average $\gamma, \gamma_{t h}$ is the threshold value, $F$ denotes the cumulative distribution function (CDF) of $\gamma$, and $T_{\text {put,max }}$ denotes the maximum data rate. A detailed description of the throughput model can be found in [12]. We define the relative throughput as $T_{\text {put }} / T_{\text {put,max }}$ and use the relative throughput hereafter. Note that the relative throughput can be regarded as the probability of detecting multiple streams.

For simplicity, we assume open-loop MIMO systems with $\mathrm{ZF}$ receivers and that transmit antennas are uncorrelated. The Kronecker channel model [1] is used:

$$
\mathbf{H}=\mathbf{R}^{1 / 2} \mathbf{H}_{w}
$$

where $\mathbf{H}_{w}$ denotes the spatially white MIMO channel with i.i.d. and unit variance complex Gaussian variables, $\mathbf{R}$ is the correlation matrix at the receive side, and $\mathbf{R}^{1 / 2}$ is the Hermitian square root of $\mathbf{R}$. For simplicity and without loss of generality, we assume unity input signal power and noise variance, the SNR of the $i$ th stream is then [1]

$$
\gamma_{i}=1 /\left[\left(\mathbf{H}^{H} \mathbf{H}\right)^{-1}\right]_{i, i}
$$

where $[\mathbf{X}]_{i, i}$ denotes the $i$ th diagonal element of the matrix $\mathbf{X}$.

Fig. 3 shows the relative throughputs of $4 \times 2$ and $4 \times 4$ openloop MIMO systems with ZF receivers supporting 2 and 4 data streams with and without correlation, respectively.

We see that a small uniform correlation of -0.3 degrades the $4 \times 2$ throughput by about $1 \mathrm{~dB}$ (i.e., it needs $1 \mathrm{~dB}$ more power to achieve the same throughput as in i.i.d. channel case); but the same correlation of -0.3 will degrades the $4 \times 4$ MIMO systems by about $5 \mathrm{~dB}$. On the other hand, a uniform correlation of the same magnitude but with opposite phase, i.e., 0.3 , results in negligible throughput performance degradations. This can be explained intuitively: the eigenvalues of the correlation matrix with uniform correlation of -0.3 are $1.3,1.3,1.3$, and 0.1 , whereas the eigenvalues of the correlation matrix with uniform correlation of 0.3 are 1.9, $0.7,0.7,0.7$; and it is the smallest eigenvalue of 0.1 that makes it difficult to detect the fourth data stream, implying a worse throughput performance for the $4 \times 4$ MIMO system with full spatial multiplexing. For the $4 \times 2$ MIMO system with full spatial multiplexing, the throughput performance degradation of the uniform correlation of -0.3 is not severe because the best two eigenvalues ensure the reliable transmission of the two streams.

Comparing Figs. 2 and 3, it seems that the correlation (e.g., the uniform correlation of -0.3) may have a much more profound effect on the throughput performance than that on the diversity performance. This is because it suffices to have one large enough eigenvalue for the diversity techniques to work (since the diversity techniques handle only one stream); for the spatial multiplexing, it is vital to have $M$ strong enough eigenvalues in order to support the transmission of $M$ streams.

\section{Capacity}

Unlike throughput, capacity is the theoretically maximum achievable data rate. To achieve the capacity, the input signal needs to be Gaussian distributed [14] and the MCS should have an infinitesimal granularity in order to support any data rate that is matched with the fading channel. As a result, capacity is usually not achievable in practical systems. Nevertheless, we still characterize the MIMO system using it in this paper, because it is the most popular MIMO performance metric, e.g., [1]-[5].

The instantaneous capacity (or mutual information) of a open-loop MIMO system is [1]

$$
C=\log _{2}\left[\operatorname{det}\left(\mathbf{I}+\frac{\gamma_{0}}{N_{t}} \mathbf{H} \mathbf{H}^{H}\right)\right]
$$

where $\mathbf{I}$ is the identity matrix, det denotes the determinant, $\log _{2}$ represents the logarithm to the base $2, \gamma_{0}$ is the average SNR per receive antenna, and $\mathbf{H}$ is given by (9). 

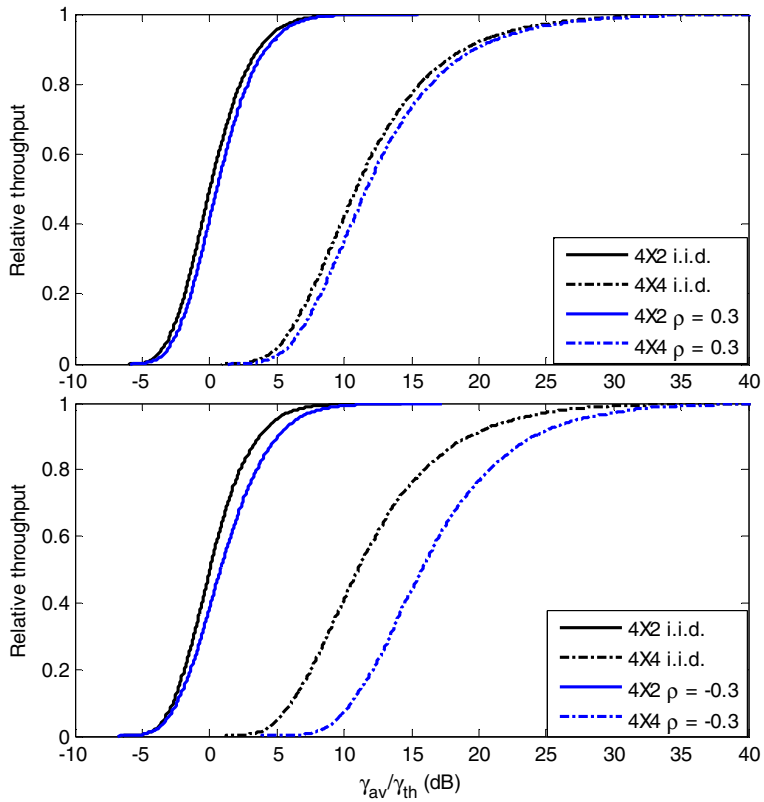

Fig. 3. Relative throughput with and without correlation for $4 \times 2$ and $4 \times 4$ MIMO systems supporting 2 and 4 data streams, respectively. The upper graph corresponds to the uniform correlation of 0.3 ; the lower graph corresponds to the uniform correlation of -0.3 . Note that for a fixed MCS the (absolute) throughput of the $4 \times 4$ system is twice of that of the $4 \times 2$ system. However, their relative throughputs (i.e., probabilities of detecting 4 and 2 data streams) are both normalized to one.
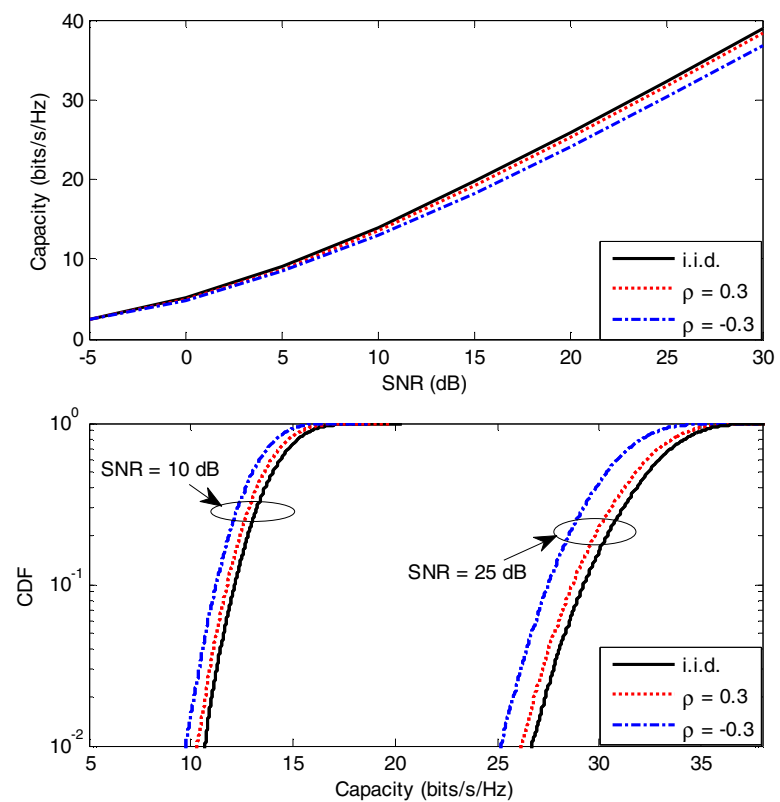

Fig. 4. Ergodic capacity (upper) and CDF of the instantaneous capacity (lower) of a $4 \times 4$ open-loop MIMO system.
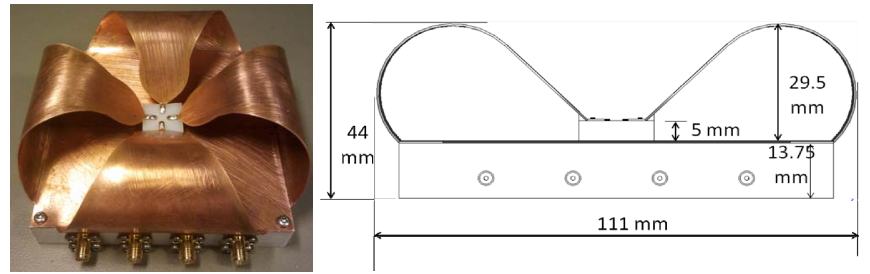

Fig. 5. Photo (left) and profile drawing (right) of the bowtie antenna [15].
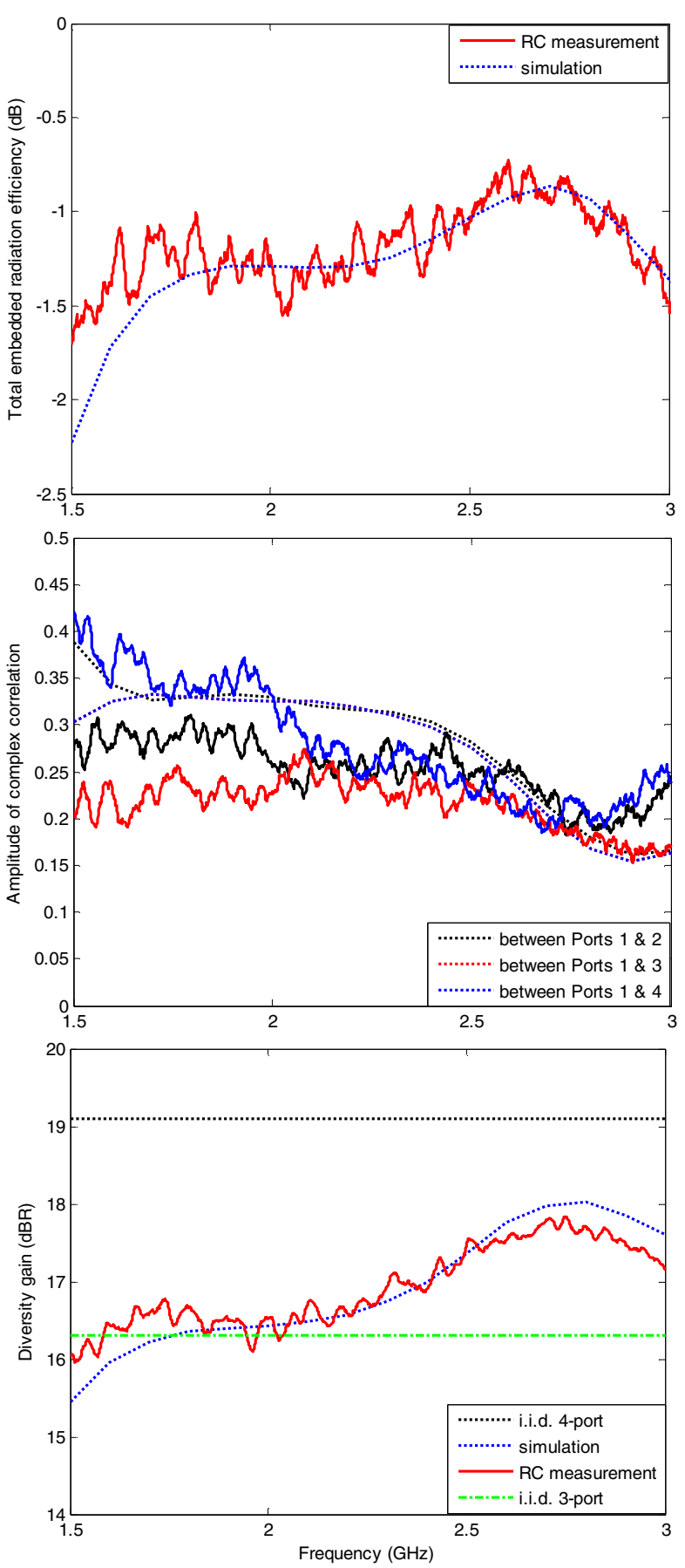

Fig. 6. Embedded radiation efficiency (top), correlation magnitude (middle), and MRC diversity gain (bottom) of bowtie antenna.

Fig. 4 shows the ergodic capacity (i.e., the average of the instantaneous capacity) as well as the CDF of the instantaneous capacity. As can be seen, the uniform correlation of -0.3 yields a worse MIMO capacity performance than that of the uniform correlation of 0.3 ; and the difference is more noticeable at high SNR. Note that the capacity-achieving system allows adaptive transmission of arbitrary streams according to the instantaneous channel condition, e.g., in a rank-deficient MIMO channel the capacity 
is achieve by a combination of spatial multiplexing and diversity by transmitting less than four streams (each stream with arbitrary payload). Therefore, the complex correlation effect on the capacity is less profound than that on the throughput with fixed MCS and number of streams (cf. Section II-B). Nevertheless, it is shown again that the phase of the correlation affects the MIMO performance.

\section{Interpretation of Phase of Correlation}

It is impossible to change the phase of the uniform correlation (and therefore the MIMO performance) by adding half-wavelength cables to some of the antenna ports. If we add half-wavelength cables to all the antenna ports, the correlation phase will be unchanged. And adding a half-wavelength cable to the first port of a multi-port antenna will only change the phases of the correlations in the first row/column of the correlation matrix by $180^{\circ}$ (resulting in a non-uniform correlation matrix). In such case the eigenvalues (and therefore MIMO performance) remain the same. We assume in this work that it is possible to find two different multi-port antennas with uniform yet out-of-phase correlation matrices that are positive semi-definite [9] for physical reasons.

\section{REVERBERATION CHAMBER MEASUREMENT}

In order to further disprove the common belief that a correlation with 0.5 or smaller magnitude is negligible for diversity gain, we performed reverberation chamber (RC) measurement [7] of a compact wideband 4-port bowtie antenna [15].

Fig. 5 shows a photo and a profile drawing (with dimensions) of the bowtie antenna. It was designed to cover the frequency range of $1.5 \sim 3 \mathrm{GHz}$. Fig. 6 shows its total embedded radiation efficiency, correlation magnitude, and MRC diversity gain, respectively. The simulation curves in Fig. 6 are obtained based on simulated embedded radiation patterns, embedded radiation efficiency, and numerically generated i.i.d. channel. There are good agreements between the RC measurements and simulations. Note that the simulated correlations (based on the CST simulated embedded radiation patterns [16]) between any two ports are approximately -0.3 . Also note that due to manufacture tolerance and measurement uncertainty (i.e., it is difficult to measure small correlation accurately) the measured correlations slightly differ from the simulated ones. It can be seen from Fig. 6 that the smaller correlations $(-0.3)$ cause about $1.5 \mathrm{~dB}$ degradation of the diversity gain in addition to the efficiency degradation. Hence, when the number of antenna ports is larger than two, even small correlations may not be negligible.

\section{CONCLUSIONS}

This work revisits the complex correlation and its effect on MIMO performances. In contrast to the common beliefs that the correlation magnitude of 0.5 is negligible for MIMO performance and the correlation affects the MIMO performance only via its magnitude, it is found in this work that the latter is only true for two-port antennas and, in general, the correlation effect on the MIMO performances depends on its phase and the number of antenna ports. For an antenna with many ports, even a small correlation may not be negligible. Correlations with the same magnitudes but different phases may result in different MIMO performances, especially for the spatial multiplexing throughput.

\section{REFERENCES}

[1] A. Paulraj, R. Nabar and D. Gore, Introduction to space-time wireless communication, Cambridge University Press, 2003.

[2] C. Waldschmidt, S. Schulteis and W. Wiesbeck, "Complete RF system model for analysis of compact MIMO arrays," IEEE Trans. Veh. Technol., vol. 53, pp. 579-586, May 2004.

[3] J. W. Wallance and M. A. Jensen, "Mutual coupling in MIMO wireless systems: a rigorous network theory analysis", IEEE Trans. Wireless Commun., vol. 3, no. 4, pp. 1317-1325, July 2004.

[4] J.-Y. Chung, T. Yang, and J. Y. Lee, "Low correlation MIMO antennas with negative group delay," Progress In Electromagnetics Research C, vol. 22, 151-163, 2011.

[5] G. Levin and S. Loyka, "From multi-keyholes to measure of correlation and power imbalance in MIMO channels: outage capacity analysis," IEEE Trans. Inf. Theory, vol. 57, no. 6, pp. 3515-3529, Jun. 2011.

[6] M. Schwartz, W. R. Bennet, and S. Stein, Communication Systems and Techniques. IEEE press, 1996.

[7] P.-S. Kildal and K. Rosengren, "Correlation and capacity of MIMO systems and mutual coupling, radiation efficiency and diversity gain of their antennas: Simulations and measurements in reverberation chamber", IEEE Commun. Mag., vol. 42, no. 12, pp. 102-112, Dec. 2004.

[8] W. C. Y. Lee, "Mutual coupling effect on maximum-ratio diversity combiners and application to mobile ratio," IEEE Trans. Commun. Technol., vol. COM-18, pp. 779-791, Dec. 1970.

[9] A. J. Laub, Matrix Analysis for Scientists and Engineers. Philadelphia, PA: SIAM, 2005.

[10] X. Chen, P.-S. Kildal, J. Carlsson, and J. Yang, "MRC diversity and MIMO capacity evaluations of multi-port antennas using reverberation chamber and anechoic chamber," IEEE Trans. Antennas Propag, vol. 61, no. 2, pp. 917-926, Feb. 2013.

[11] E. Jorswieck and H. Boche, "Majorization and Matrix-Monotone Functions in Wireless Communications," Foundations Trends Commun. Inf. Theory, vol. 3, no. 6, pp. 553-701, 2006.

[12] P.-S. Kildal, A. Hussain, X. Chen, et al., "Threshold receiver model for throughput of wireless devices with MIMO and frequency diversity measured in reverberation chamber," IEEE Antennas Wireless Propag. Lett, vol. 10, pp. 1201-1204, 2011.

[13] X. Chen, P.-S. Kildal, and M. Gustafsson, "Characterization of Implemented Algorithm for MIMO Spatial Multiplexing in Reverberation Chamber," IEEE Trans. Antennas Propag., vol. 61, no. 8, pp. 4400-4404, Aug. 2013.

[14] T. M. Cover and J. A. Thomas, Elements of Information Theory. John Wiley \& Sons, 1991.

[15] H. Raza, A. Hussein, J. Yang, and P.-S. Kildal, "Wideband Compact 4port Dual Polarized Self-grounded bowtie Antenna", submitted to IEEE Trans. Antennas Propag., Dec. 2013.

[16] R. G. Vaughan and J. B. Andersen, "Antenna diversity in mobile communications", IEEE Trans. Vehic. Technol. vol. 36, no. 4, pp. 149172, Nov. 1987. 